\title{
Expressive aphasia: reporting a phonological retrieval hypothesis for auditory verbal memory deficits
}

\begin{abstract}
Studies have pointed to a possible close relationship between language deficits and impairment in verbal auditory memory in aphasia. Additionally, there is evidence that aphasics who have predominantly semantic deficits have greater impairment in immediate word recall tasks. These studies may contribute for an understanding of the role of auditory verbal memory in language processing. They may also shed light into the nature of verbal memory deficits, showing whether or not they are predominantly semantic or phonological. In the present case report, we present the word recall performances of two chronic predominantly expressive aphasic cases, one with more phonological than semantic deficits and the other with exclusively phonological deficits. The language profile of these patients show relatively preserved semantic processing and predominance of lexical retrieval and phonological disorders. The results are discussed in terms of pointing for the role of verbal memory in the access to phonological representations. Additionally, the report draws attention for considering data from late recall and word recognition skills, as well as working memory skills in aphasia. These findings allow the consideration of the role that executive retrieval skills may play in the access to phonological representations of words.
\end{abstract}

Volume 7 Issue 3 - 2017

\author{
Camila Leonhardt Grigol,' Magda Aline \\ Bauer,' Lenisa Brandão² \\ 'Programa de Reabilitação em Linguagem e Cognição (RELINC, \\ CIPAS), Universidade Federal do Rio Grande do Sul, Brazil \\ 2Departamento de Saúde e Comunicação Humana, Universidade \\ Federal do Rio Grande do Sul, Brazil
}

\begin{abstract}
Correspondence: Lenisa Brandão, Professor of the Departament of Health and Human Communication, Instituto de Psicologia - Universidade Federal do Rio Grande do Sul, Rua Ramiro Barcelos, 26, Bairro Santa Cecília, Porto Alegre - RS Brazil, CEP: 935-3, Fax (55)(5I) 991865323 ,

Email lenisabrandao@hotmail.com
\end{abstract}

Received: June 26, 2017| Published: August 23, 2017

Keywords: aphasia, working memory, word recall, language, processing

Abbreviations: ID, working memory inverse digit ordering span task; WS, working memory word and sentence span task; IR, verbal memory immediate word recall task; LR, verbal memory late word recall task; $\mathrm{R}$, verbal memory word recognition task

\section{Introduction}

The nature of the relationship between language, working memory and auditory-verbal memory seems to remain unclear and classical language and memory models appear to view these skills as completely separate modules. Language processing models consider the existence of devices or buffers which store linguistic information. Levelt ${ }^{1}$ describes a short-term memory device called the articulatory buffer in which a phonetic plan is temporarily stored to be articulated. Nickels ${ }^{2}$ also describes the auditory processing of words with a model that contains phonological input and output buffers, as well as phonological input and output lexicons. Dell's interactive activation model for word retrieval postulates that lexical retrieval requires a stage of lexical access and a phonological stage. In the lexical stage, the semantic concepts would activate corresponding lexical representations and, in the phonological stage, the lexical representations would send activation to phonological representations, then the sounds corresponding to the lexical item would be selected. Failures of activation between these interactive stages would explain lexical and phonological difficulties. ${ }^{3}$

Although language models suggest the existence of linguistic storage devices, they do not consider working memory or auditory short-term memory as components of language processing. On the other hand, memory models also consider the storage of linguistic information and do not take into account the storage devices described in language processing models. The classical working memory model ${ }^{4}$ includes a phonological loop that would store numbers and words while performing tasks. According to this model, lists of items would be temporarily stored in auditory-verbal memory via a phonological code. This idea is supported by studies with typical speakers, which demonstrate phonological similarity and word size effects. ${ }^{5}$
According to Martin et al., ${ }^{6}$ explanations about the process of temporary linguistic activation would encompass the functioning of auditory-verbal memory. Therefore, the ability to store words temporarily, that is, auditory-verbal memory, would be a property of language processing, that is, auditory-verbal memory capacity would be required in language processing. This idea is consistent with evidences of overlapping deficits in patients with neurological lesions, markedly aphasic patients. ${ }^{5}$

Aphasia is a language disorder caused by injury to brain regions important to language processing, being characterized by expressive and or comprehensive deficits. However, not only cognitive functions well known as linguistic are impaired in aphasia. Several cognitive abilities, such as memory, may be affected in this population. ${ }^{7}$ In addition, increasing evidence shows that many cognitive components are recruited and used in varying extents during the communication process. $^{8}$

A recent review of the literature ${ }^{5}$ shows that aphasic patients seem to present a severe difficulty in word recall tasks. This confirms a very close relationship between language deficits and impairment in verbal auditory memory. Traditionally, aphasics have lower immediate word spans when compared to individuals with left hemisphere lesions who do not present aphasia and when compared to individuals with right hemisphere lesions. Thus, verbal auditory memory capacity measured by immediate recall is reduced not only because of slower auditory processing caused by a brain injury, but because word recall is possibly a skill which takes part in language processing areas affected in aphasia. ${ }^{9}$

Studies that seek to go beyond the identification of verbal auditory memory reduction in aphasics investigate the auditory memory deficits that appear in this population and compare these deficits with the types of linguistic deficits observed in aphasia. Identifying whether the linguistic deficits of aphasics are predominantly semantic or phonological may help understand the nature of the relationship between verbal memory and language processing. ${ }^{5}$ Until now, evidence shown points to the idea that aphasics who have predominantly 
semantic deficits have greater impairments in immediate word recall tasks. According with these findings, auditory verbal memory deficits in aphasia would have a semantic nature.

In the present case report, we focus on word recall and working memory performances of two chronic predominantly expressive aphasic cases, one with lexical retrieval deficits characterized mostly by difficulties in the phonological level and the other with exclusively phonological deficits. In addition to immediate word recall, we include data from late recall, word recognition, and working memory tasks in order to consider the hypothesis that deficits in the recovery of phonological representations of words may be influenced by an executive retrieval component of verbal memory.

\section{Case presentation}

\section{Case A}

This participant is a right-handed 51 years old female patient, who had 6 years of formal education. She suffered an ischemic stroke a year from the time she took this evaluation and started treatment. After stroke, she started complaining of hearing difficulties. Her hearing was assessed, but her auditory thresholds were well within normal limits. The difficulties presented by this patient demonstrate deficits in auditory processing, with a mild deficit in repeating words and sentences. Naming skills and word comprehension are preserved when assessed by subtests of the Boston battery. However, phrase comprehension is mildly impaired. Her spontaneous discourse is rich in phonemic and morphemic paraphasia occurrences, while episodes of neologism and anomia are also observed. Semantic paraphasia is not present in the discourse context, neither does it appear in formal testing. Language profiles were obtained in both cases with the Boston Battery. ${ }^{10}$ The neuropsychological profile obtained through NeupsilinAf shows impaired working memory, as well as deficits in immediate and late word recall subtests. She recovers well with cues in the word recognition task and shows preserved attention, perception, semantic and visual memory skills.

\section{Case B}

This participant is a male, aged 61 years old, with 11 years of formal study, and right-hand dominance. He had an ischemic stroke 3 years before he undertook this evaluation and started treatment. The difficulties presented by this patient demonstrate severe deficits in word retrieval, with low scores in the Boston naming task as well as a great number of anomia episodes in spontaneous discourse. He also has a mild deficit in repeating words and sentences, as well as in understanding words and sentences. His spontaneous discourse is marked by the severity of anomia and apraxia symtoms. Hesitations, as well as sylable and word repetition qualify his discourse as very disfluent. No episodes of semantic or phonemic paraphasia are observed in the discourse context, neither do they appear in formal testing. Some morphemic paraphasia typical of agrammatism is observed. His neuropsychological profile shows even lower scores in working memory, immediate and late word recall subtests when compared to Case A. In the word recognition task, he also recovers well with the word cues. Similar to Case A, he also shows preserved attention, perception, semantic and visual memory skills. Figure 1 shows a summary of the neuropsychological profiles of both cases. Neuropsychological functions were tested with the Neupsilin-Af, an instrument that is adapted for the cognitive testing expressive aphasics.

As shown in the graphics above, both cases have verbal and working memory deficits. In addition, both benefited from word cues in the recognition task, which shows that their impairment is predominantly in the retrieval of words and not in their storage. Case A has a better performance in working memory and word recall tests than Case B. Particularly in the late recall task, Case B shows severe impairment, with no recollection of words.
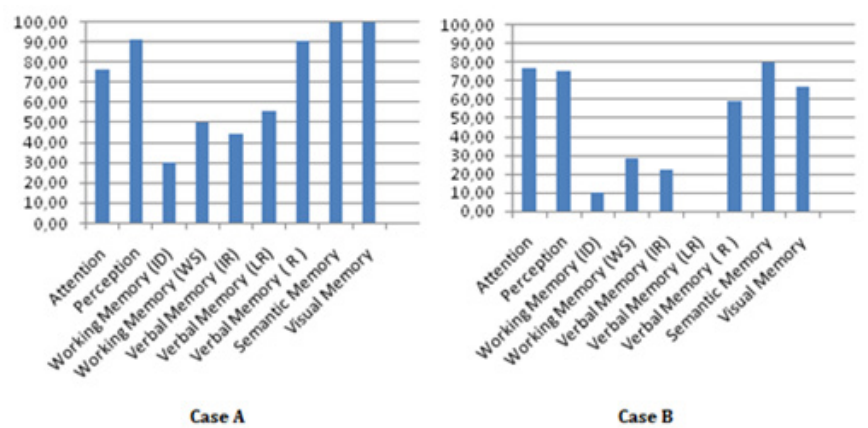

Figure I Cases on Ischemic stroke.

If we consider the language profile differences, Case B has greater deficits in classical linguistic tasks. Working memory and word recall tasks follow this impairment. The fact that he has severe anomia in spontaneous discourse as well as naming deficits point for a rupture of the phonological output lexicon. Performance on the recognition task also speaks for a phonological retrieval rather than a semantic impairment. However, his deficits are not exclusively phonological and do include semantic difficulties (comprehension problems). This finding does not rule out the role of lexical semantics as an explanation for the severity of his verbal and working memory deficits. However, it is clear that semantics alone does not explain the low performance of Case B in the memory tasks. Case A shows clearly a picture of phonological auditory deficits which cannot be related to semantic impairment. Although less severe, her verbal memory and working memory difficulties also contrast with the preservation of the rest of the neuropsychological functions. Thus, it seems plausible to hypothesize that Case A's phonological impairments alone may relate to her word recall and working memory deficits.

\section{Discussion}

In the last 20 years aphasia research has corroborated to the idea that the problem that characterizes aphasia is a difficulty in accessing linguistic representations rather than a loss of linguistic representations. A paradigm shift is occurring in the field of aphasiology: researchers are beginning to speak not in terms of cognition and language, but in terms of the cognitive requirements of language processing. This paradigm shift implies viewing some of the main aphasic deficits as the result of impairment in the access to linguistic representations. ${ }^{5}$

In this context, working memory and auditory-verbal memory are considered as important cognitive functions which possibly take part in language processing. When preserved, these functions may facilitate the access to linguistic representations. Word recall may depend not only on memory storage, it may also relate to components required from working memory. The latter involves both temporary storage systems and an executive control device that provides the capacity to actively manipulate stored information. Recently, functional neuroimaging findings show that working memory tasks activate the Broca area. ${ }^{11}$ These findings corroborate with the idea that working memory has a significant role in the linguistic system.

A recent review of the literature ${ }^{5}$ shows that studies on aphasia and episodic verbal memory have pointed to a close relationship between the predominance of semantic deficits and impairment of 
verbal auditory memory. In the present case report, we demonstrated that phonological deficits may also relate to the verbal and working memory deficits of aphasics. Our report is not alone in this framework. Almost twenty five years ago, Trojano et al., ${ }^{12}$ described a case who could repeat words but not nonwords. He had no difficulties in reading both words and nonwords, so his impairment was related to auditory phonological coding, characterizing a picture of phonemic deafness. The authors concluded with the short-term memory and verbal learning performance of this case that he had a selective short-term memory defect related to auditory phonological coding as well as short-term memory capacity limitations. ${ }^{13}$

The knowledge we have today about language and communication processing demonstrates that we can no longer separate language and cognition fields. This has important implications not only for theoretical models, but especially for establishing the interdisciplinary nature of neuropsychology of language and communication in clinical practice. The assessment of cognitive skills related to language processing and communication is fundamental for the effectiveness of speech-language therapy. For a long time many cognitive functions were not classically considered as important in the understanding of communication disorders. As science progresses, models are being reviewed and some skills (such as auditory verbal memory and working memory) are being directly linked to language processing. As ethics and client centered perspectives postulate, clinical practice should follow scientific progress..$^{14,15}$

\section{Conflicts of interest}

None.

\section{Funding}

None.

\section{References}

1. Willem JM Levelt. Speaking: From Intention to Articulation. The American Journal of Psychology. 1990;103(3):409-418.

2. Nickels L, Stremmer B, Whitaker H. The Hypothesis testing approach to the assessment of language. The Handbook of Neuroscience of Language. 2008;13-22.
3. Dell GS, Schwartz MF, Martin N, et al. Lexical access in aphasic and nonaphasic speakers. Psychol Rev. 1997;104(4):801-838.

4. Baddeley A. The articulatory loop. Working memory. 1986;75-101.

5. Minkina I, Rosenberg S, Kalinyak-Fliszar M, et al. Short-Term Memory and Aphasia: From Theory to Treatment. Semin Speech Lang. 2017;38(1):17-28.

6. Martin N, Ayala J, Saffran EM. Lexical influences on serial position effects in verbal STM span in aphasia. Brain and Language. 2002;83(1):92-95.

7. Hillis AE. Aphasia progress in the last quarter of a century. Neurology 69(2): 200-213.

8. Helm-Estabrooks N (2002) Cognition and aphasia: A discussion and a study. J Commun Disord. 2007;35(2):171-186.

9. Martin N. Word processing and verbal short-term memory: How are they connected and why do we want to know. Brain Lang. 2000;71(1):149-153.

10. Harold Goodglass, Edith Kaplan, Barbara Barresi. Boston Diagnostic Aphasia Examination. ( $3^{\text {rd }}$ edn), (BDAE-3). 2001.

11. Paulesu E, Shallice T, Danelli L, et al. Anatomical Modularity of Verbal Working Memory? Functional Anatomical Evidence from a Famous Patient with Short-Term Memory Deficits. Front Hum Neurosci. 2017;11:231.

12. Trojano L, Stanzione M, Grossi D. Short-term memory and verbal learning with auditory phonological coding defect: A neuropsychological case study. Brain Cogn. 1992;18(1):12-33.

13. Potagas C, Kasselimis D, Evdokimidis I. Short-term and working memory impairments in aphasia. Neuropsychologia. 2011;49(10):2874-2878.

14. Radanovic M, Mansur LL, Scaff M. Normative data for the Brazilian population in the Boston Diagnostic Aphasia Examination: influence of schooling. Braz J Med Biol Res. 2004;37(11):1731-1738.

15. Fontoura DR, Rodrigues JC, Fonseca RP, et al. Adaptação do Instrumento de Avaliação Neuropsicológica Breve NEUPSILIN para avaliar pacientes com afasia expressiva: NEUPSILIN-Af. Ciências \& Cognição. 2011;16(3):78-94. 